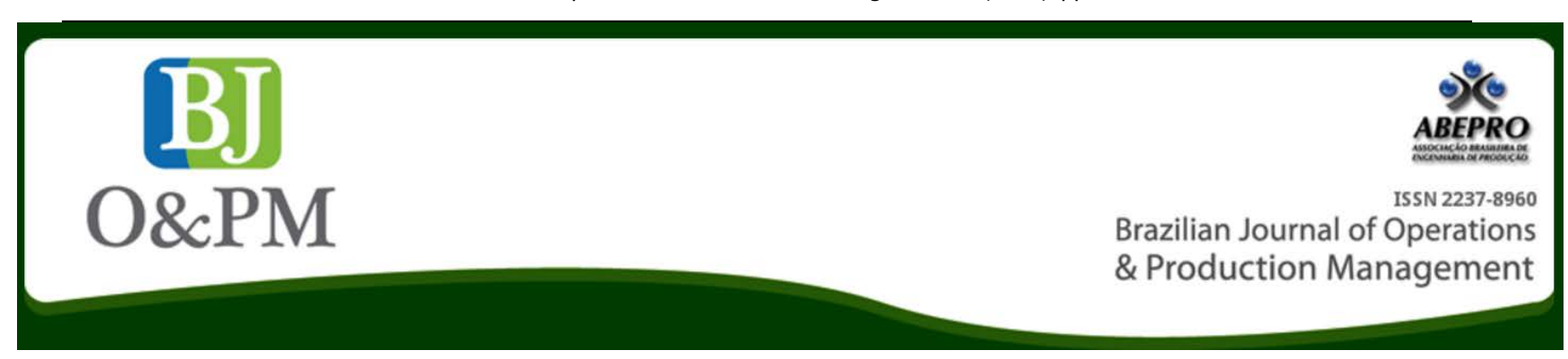

\title{
ON THE HOTELLING'S T, MCUSUM AND MEWMA CONTROL CHARTS' PERFORMANCE WITH DIFFERENT VARIABILITY SOURCES: A SIMULATION STUDY
}

\author{
D. A. O. Moraesa ; F. L. P. Oliveira ${ }^{\text {b }}$ L. H. Duczmal ${ }^{c}$ \\ ${ }^{\text {a }}$ Federal University of Santa Maria (UFSM) - Santa Maria, RS, Brazil \\ ${ }^{b}$ Federal University of Ouro Preto (UFOP) - Ouro Preto, MG, Brazil \\ ${ }^{c}$ Federal University of Minas Gerais (UFMG) - Belo Horizonte, MG, Brazil
}

\begin{abstract}
This work is a simulation study to investigate the sensitivity of multivariate control charts for monitoring mean vectors in a bivariate Gaussian process with individual observations. The multivariate cumulative sum (MCUSUM), the multivariate exponentially weighted moving average (MEWMA) and Hotelling's T charts are selected for analysis due to their common dependency on the non-centrality parameter. The chart performance is evaluated through the average run length (ARL) or the average time to signal. The impact of utilising in-control limits computed from known parameters or Phase I sample estimates is considered for mean vector shifts. Although designed to monitor mean vectors, the sensibility of the control charts is additionally analysed through different variability sources, including the mixing effect of mean vector shifts with increasing variances or positive autocorrelation in the out-of-control process.
\end{abstract}

Keywords: Quality control, Mean vectors, Non-centrality parameter, Average run length, Increasing variances, Increasing autocorrelations.

\section{INTRODUCTION}

Developed to study the influence of social castes in India in the early $20^{\text {th }}$ century, the Mahalanobis distance (Mahalanobis, 1936) is an important example of a dissimilar metric in various disciplines. Among many existing applications of this distance, in the field of statistical process control (SPC) it is known as the non-centrality parameter. With the increase in computational power over the last century and the growing number of applications, the Monte Carlo method can help researchers to understand this distance behaviour under different simulated scenarios.

In SPC, the non-centrality parameter is frequently applied in control charts to detect process changes, triggering a signal as soon as the underlying process shifts from the in-control state to the out-of-control state. To evaluate the control chart performance, the metric typically adopted is the average run length (ARL) or average time to signal (ATS). The ATS is the process ARL when the time interval between samples is fixed at one time unit, as during this simulation study.

ABEPRO

DOI: 10.14488/BJOPM.2015.v12.n2.a2
An important factor for rapid change detection is selecting the correct method, which depends on the available data and the change to be monitored. Montgomery (2001) elaborates on the decision schemes recommended to correctly choose a control chart method. Lowry and Montgomery (1995) present an additional review. Although the multivariate exponentially weighted moving average (MEWMA) of Lowry et al.,(1992) and the multivariate cumulative sum (MCUSUM) charts of Crosier (1988) are popular and more suitable to detect small changes, the $\mathrm{T}^{2}$ chart is suggested to monitor the mean vector for large-scale shifts. Mahmoud et Maravelakis $(2010,2011)$ estimate the parameters for evaluating the performance of two types of MCUSUM and MEWMA control charts. These charts are directly based on the non-centrality parameter and can be applied to a number $p$ of variables, where $p \geq 1$. When $p=1$, all methods are reduced to their respective univariate schemes, which are the CUSUM, EWMA and X control charts. Recent work proposes several chart modifications, such as the double exponentially weighted moving average (dEWMA) method proposed by Alkatani et Schaffer (2012).

To monitor the covariance matrix, Montgomery (2001) 
recommends the moving range and generalised variance tests, Riaz et Does (2008) suggest utilising supporting information and Costa and Machado (2008) postulate the VMAX procedure. Yeh- et al.,(2006) proposed modifications of the EWMA method based on the generalised variance.

The approaches for simultaneously monitoring changes in the mean vector and the covariance matrix are numerous, and we highlight the integration of the exponentially weighted moving average procedure with the generalised likelihood ratio test of Zhang et Wang (2010) and Khoo et al., (2010), whose statistics are based on the maximum of the absolute values of the two dEWMA statistics, one of which controls the mean vector and the other the covariance matrix.

The numerous proposals to monitor changes in the mean vector of autocorrelated data (Montgomery, 2001) include traditional methods that fit the time series and subsequently implement control techniques on the model residuals produced by the fit.

Although designed to monitor mean vector shifts, the present study analyses the sensibility of the MCUSUM, MEWMA and Hotelling's T statistics for process changes in the mean vector with different sources of variation. First, the chart performance for mean vector shifts is compared considering known parameters or training on small samples sizes. The simulated mean vector shifts includes comparisons about the shift's direction in correlated and non-correlated processes with known parameters. Increasing variances and the influence of a vector autoregressive model are additionally measured and analysed using the ARL value, while the mean vector is fixed.

These two variables are chosen to analyse the multivariate point process for their broad application in space-time problems and to provide a general example of multivariate data. Another positive aspect of studying only two variables is the potential to visually identify the in- and- out-ofcontrol observations in their original spaces with threedimensional observations to make more direct conclusions and facilitate an understanding of what occurs in the higherdimensional spaces. To emphasise this objective, Lowry et Montgomery (1995) recommend extending additional graphical approaches, including the Polyplot method (Blazek et al., 1987), beyond the Hotelling's T statistic and including techniques, such as the MEMWA chart, that effectively detect small changes. Although different methodologies for data visualization are employed in conjunction with the control charts, this work serves as a tool for a continuous data view in the scatter-plots, estimating confidence ellipses for the current process based on principal component analysis (PCA).
In Section 2, we present the control charts methodology with a review of the non-centrality parameter, which is the common basic distance among the Hotelling's T, MCUSUM and MEWMA control charts. Additionally we provide a brief explanation of PCA as a tool for data visualisation in the scatter-plots. Section 3 analyses different experiments for shifts in the mean vector with known parameters or sample estimates. Because inertia problems may occur with non-Shewhart control charts, we demonstrate our recommendation for the simultaneous monitoring of a non-Shewhart and Shewhart-type control charts to avoid detection delay. Further, the ARLs measures the chart sensibility due to increasing variances, autocorrelation, and the resulting performance of mixing those effects with mean vector shifts. The final section discusses the control charts performance and sensibility in the proposed scenarios and prospects for future work.

\section{CONTROL CHARTS METHODOLOGY}

In the general case, suppose that vectors $\mathbf{x}_{1}, \mathbf{x}_{2}, \mathbf{x}_{3}, \ldots$ of dimension $p \times 1$ represent sequential observations of $p$ characteristics over time. The observations $\mathbf{x}_{i}, i=1,2, \ldots$ , are assumed to be independent random vectors of a multivariate normal distribution with a mean vector $\mathbf{M}_{0}$ and covariance matrix $\Sigma_{0}$. Without loss of generality, consider that $\mathbf{M}_{0}=(0,0, \ldots, 0)^{\prime}=\mathbf{0}$ and $\boldsymbol{\Sigma}_{0}=\mathbf{I}$.

The three control charts analysed share the property that their performances, as measured by ARL, depend on

$\mathbf{M}$ and $\boldsymbol{\Sigma}_{0}$ only according to the value of the noncentrality parameter (Lowry et al., 1992), which is given by

$$
d=\left(\mathbf{M}^{\prime} \boldsymbol{\Sigma}_{0}^{-1} \mathbf{M}\right)^{1 / 2} \text {. }
$$

When $\boldsymbol{\Sigma}_{0}$ is the identity matrix, $d$ is reduced to a Euclidean distance. In his original formulation, Hotelling suggests the utilisation of $d^{2}$ to avoid the labor of extracting the square root. If the in-control process is not symmetrical around its centre of mass, as occurs for correlated variables, the Euclidean distance does not consider the process covariance, thus making it directionally dependent. To quantify the magnitude of shifts without directional dependence, the shifts should be correctly weighted by the covariance matrix. The statistical pattern recognition literature (Therrien, 1989) shows that the non-centrality parameter, also known as the Mahalanobis distance, is related to other statistical measures known as the Divergence $(D)$ and Bhattacharyya $(B)$ 
distances. When the covariance matrices of two processes are equal, the relationship $d^{2}=D=8 B$ is valid.

In classification problems, those distances are present in single and composite tests. For the composite problem, each observation is compared within two or more classes and attributed to the one with the shortest distance. The non- centrality parameter in a control chart evaluates the relative vector distance to the in-control process mean. Thus, problems related to the control charts often arise as single hypothesis tests, i.e., when the unique known class is the in-control state. The analysis of signals from radar devices is an example of single hypothesis tests applied in the pattern recognition field, when the aim is the simple recognition of potential targets.

Note the existence of two implicit assumptions in the performance comparisons based on the non- centrality parameter. First, any shift, regardless of size, must be detected as early as possible. Second, a shift from $\mathbf{M}_{0}$ to $\mathbf{M}_{1}$ is detected as quickly as a shift from $\mathbf{M}_{0}$ to $\mathbf{M}_{2}$ if $\mathbf{M}_{1}^{\prime} \mathbf{\Sigma}_{0}^{-1} \mathbf{M}_{1}=\mathbf{M}_{2}^{\prime} \boldsymbol{\Sigma}_{0}^{-1} \mathbf{M}_{2}$. As the ARL value is a function of the non-centraility parameter $d$, the comparisons between the methods are simplified with analysis of the curve ARL vs. $d$. Alternatively, if the charts do not share this property, their relative performance may vary depending on

$\boldsymbol{\Sigma}$, i.e., even for a given matrix $\boldsymbol{\Sigma}$, a chart may more effectively detect changes in some directions and less effectively in other directions.

\section{HOTELLING'S T CONTROL CHART}

The statistic proposed by Hotelling (1947), as defined in equation (2), triggers a signal when there is a significant shift in the mean vector, such that

$$
\mathrm{T}_{i}=\left[\left(\mathbf{x}_{i}-\mathbf{M}_{0}\right)^{\prime} \boldsymbol{\Sigma}_{0}^{-1}\left(\mathbf{x}_{i}-\mathbf{M}_{0}\right)\right]^{1 / 2}>h_{1},
$$

where $h_{1}>0$ is the threshold specified to maintain a desired in-control average run length $\left(A R L_{0}\right)$.

There are three asymptotic distributions described in the literature to compute the in-control limits for the Hotelling's

T statistics. When we assume that the observations $\mathbf{x}_{i}$ are not time-dependent and that the process parameters are known, the $\mathrm{T}_{i}^{2}$ statistic follows a Chi-squared distribution with $p$ degrees of freedom and $h_{1}=X_{p, 1-\alpha}^{2}$ (Seber, 1984). This control chart is called a Phase II $T^{2}$-chart for individual observations with known parameters. If sample estimates $\left(\overline{\mathbf{x}}_{0}, \mathbf{S}_{0}\right)$ of $\left(\mathbf{M}_{0}, \boldsymbol{\Sigma}_{0}\right)$ are employed and $\mathbf{x}_{i}$ is an individual observation that is not independent of the estimators, then the $\mathrm{T}_{i}^{2} / d_{0}(m)$ statistic follows a Beta distribution with $p / 2 p / 2$ and $(m-p-1 / 2)$ degrees of freedom, where $d_{0}(m)=(m-1)^{2} m^{-1}$. Thus, the upper control limit is given by $h_{1}^{\prime}=d_{0}(m) B_{1-\alpha, p / 2,(m-p-1) / 2}$. This control chart is called a Phase I $T^{2}$-chart (Tracy et al., 1992). If the estimators are utilised instead of the parameters and if $\mathbf{x}_{f}$ is a future individual observation that is independent of $\left(\overline{\mathbf{x}}_{0}, \mathbf{S}_{0}\right)$, then $\mathrm{T}_{f}^{2} / d_{1}(m, p)$ follows an F-distribution with $p$ and $(m-p)$ degrees of freedom, where $d_{1}(m, p)=p(m+1)(m-1)[m(m-p)]^{-1}$

Thus, the upper control limit of this multivariate Shewhart control chart is $d_{1}(m, p) F_{1-\alpha, p, m-p}$. This control chart is called a Phase II $T^{2}$-chart with unknown parameters.

Because the multivariate Shewhart control charts only consider the information given by the current observation, they are insensitive to small and moderate shifts in the mean vector. To overcome this problem, we concisely describe the multivariate CUSUM and EWMA schemes proposed in the literature.

\section{MULTIVARIATE CUMULATIVE SUM CHART}

Among the multivariate CUSUM methods proposed by Crosier (1988), the method with the best properties in terms of performance triggers an alarm when the statistic

$$
\gamma_{i}=\left(\mathbf{s}_{i}^{\prime} \mathbf{\Sigma}_{0}^{-1} \mathbf{S}_{i}\right)^{1 / 2}>h_{2}
$$

$$
\begin{gathered}
\text { where } \mathbf{S}_{i}=\mathbf{0}, \text { if } C_{i} \leq k, \\
\mathbf{S}_{i}=\left(\mathbf{S}_{i-1}+\mathbf{x}_{i}-\mathbf{M}_{0}\right)\left(1-k / C_{i}\right) \\
\text { if } C_{i}>k, \mathbf{S}_{0}=\mathbf{0}, k>0 \text { and } \\
C_{i}=\left[\left(\mathbf{s}_{i-1}+\mathbf{x}_{i}-\mathbf{M}_{0}\right) \mathbf{\Sigma}_{0}{ }^{-1}\left(\mathbf{S}_{i-1}+\mathbf{x}_{i}-\mathbf{M}_{0}\right)\right]^{1 / 2}, \\
i=1,2, \ldots .
\end{gathered}
$$

The upper control limit $h_{2}$ is determined to provide a predefined in-control ARL by simulation. Because the ARL performance of this chart depends on the non-centrality 
parameter, Crosier recommends $k=d / 2$ for a shift detection of $d$ units.

\section{MULTIVARIATE EXPONENTIALLY WEIGHTED MOVING AVERAGE CHART}

The MEWMA method proposed by Lowry et al.,(1992) is a natural extension of the EWMA chart. Its multivariate formulation defines the EWMA vector as $\quad \mathbf{z}_{i}=\lambda \mathbf{x}_{i}+(\mathbf{I}-\lambda) \mathbf{z}_{i-1}=\sum_{j=1}^{i} \lambda(\mathbf{I}-\lambda)^{i-j} \mathbf{x}_{j}$, $i=1,2, \ldots$, where $\mathbf{z}_{0}=\mathbf{0}$, the initial in-control mean vector of the process, and $\lambda=\operatorname{diag}\left(\lambda_{1}, \lambda_{2}, \ldots, \lambda_{p}\right)$, $0 \leq \lambda_{j} \leq 1, j=1,2, \ldots, p$.

When $\lambda=\mathbf{I}$, the MEWMA control chart is equivalent to the $T$-chart. Similar to other methods, this procedure triggers an out-of-control signal when

$$
\mathrm{z}_{i}=\left[\mathbf{z}_{i}^{\prime} \mathbf{\Sigma}_{\mathbf{z}_{i}}^{-1} \mathbf{z}_{i}\right]^{1 / 2}>h_{3},
$$

where $h_{3}>0$ is chosen by simulation to obtain a predefined value of $A R L_{0}$ and $\boldsymbol{\Sigma}_{z_{i}}$ is the covariance matrix of $\mathbf{z}_{i}$. If there is no reason to differentially weigh the historical observations in the $p$ characteristics, then $\lambda_{1}, \lambda_{2}, \ldots, \lambda_{p}=\lambda$ is utilised, but when unequal weighting constants are considered, the ARL depends on the direction of the shift. The covariance matrix of $\mathbf{z}_{i}$ is calculated as

$$
\begin{gathered}
\boldsymbol{\Sigma}_{\mathbf{z}_{i}}=\sum_{j=1}^{i} \operatorname{Var}\left[\lambda(\mathbf{I}-\lambda)^{i-j} \mathbf{x}_{j}\right]=\sum_{j=1}^{i} \lambda(\mathbf{I}-\lambda)^{i-j} \mathbf{\Sigma}(\mathbf{I}-\lambda)^{i-j} \lambda ; \\
\text { when } \lambda_{1}=\lambda_{2}=\cdots=\lambda, \\
\boldsymbol{\Sigma}_{\mathbf{z}_{i}}=\left(1-(1-\lambda)^{2 i}\right) \lambda /(2-\lambda) \mathbf{\Sigma} .
\end{gathered}
$$

An approximation of the variance-covariance matrix $\boldsymbol{\Sigma}_{z_{i}}$ as $i$ approaches $+\infty$ is given as $\boldsymbol{\Sigma}_{z_{i}}=\lambda /(2-r) \boldsymbol{\Sigma}_{0}$; however, the appliance of exact variance-covariance matrix leads to a natural fast initial response (FIR) for the MEWMA chart.

\section{SCATTER-PLOTS WITH CONFIDENCE ELLIPSE ESTIMATION BY PCA}

The principal components method is a common multivariate procedure for projecting the original variable space into an orthogonal space, so less transformed variables that represent different sources of variation can be monitored together with multivariate control charts or individually with univariate control charts (Bersemis et al., 2007). Among the applications of this very useful method in multivariate quality control, Jackson (1991) studied three types of control charts based on PCA. The first type is a T control chart obtained from principal scores components, the second is a control chart for principal component residuals and the third is a control chart for each independent principal component's scores. Thus, further analysis could be made to monitor individual observations using their projections into the principal components. Bersemis et al.,(2007) offer a detailed description of multivariate process control via PCA and other projection techniques. Making a distinction between signal classification and signal representation as exposed by Fukunaga (1990), the authors applies PCA as a descriptive tool, establishing in- and- out-of-control regions for the current process and visualising them to gain an understanding of the process in conjunction with the control charts.

PCA aims to find a matrix $\mathbf{\Sigma}^{*}$ with a linear transformation of $\boldsymbol{\Sigma}$, which rotates the original axes in the directions of decreasing (or increasing) variability. In the bivariate case, the eigenvector $e_{1}=\left(e_{11}, e_{12}\right)$ associated with the first principal component $\left(\mathrm{PC}_{1}\right)$ in the rotation matrix $\boldsymbol{\Sigma}^{*}$ indicates the direction of maximum process variability, and the first eigenvalue $v_{1}$ indicates the normalised size of variation in that direction. Similarly, the second principal component $\left(\mathrm{PC}_{2}\right)$ indicates the direction and magnitude of the axis with the second most significant variability, which is orthogonal to the first one.

To plot the in- and -out-of-control ellipses in the scatter-plots showed in the present work, take the rotation angle of the estimated ellipse (in radians) with respect to the original coordinate system from the trigonometric rules, such as the arctangent of the first eigenvector, which points in the direction of greatest variability. Similarly, the second eigenvector provides the direction of the second most significant variation. The estimated axis sizes in the directions of the major and minor variability are normalised, and the eigenvalues are multiplied by the quantile of a multivariate normal distribution to establish the confidence region of $(1-\alpha) 100 \%$, where $\alpha$ is the error probability. Assuming that the subjacent process is Gaussian, we adopt $z_{(1-\alpha)}=3.023$, which corresponds to an error $\alpha=0.005$ for the estimated axis size. The in-control ellipses are drawn in blue and the out-of-control ellipse in red. 


\section{EXPERIMENTS}

As described above, the calibration of the MCUSUM and MEWMA charts to obtain a predefined value for $A R L_{0}$ involves defining the $k$-factor in the MCUSUM and $\lambda$ in the MEWMA. For the MCUSUM chart, Crosier (1988) notes that one should choose $k=d / 2$ to detect a shift with a magnitude $d$ corresponding to the noncentrality parameter. For the MEWMA method, Lowry and Montgomery (1992) illustrate optimal schemes to choose the weighting factor $\lambda$, which generally must be between 0.05 and 0.25 . The optimum suggested value of $\lambda$ to detect a unitary change in the non-centrality parameter is 0.16 .

In the present work, $\lambda=0.1$ is selected for the MEWMA chart because with the MEWMA chart using this value behaves a little better than the MCUSUM chart when $k=0.5$, which corresponds to a target shift detection of noncentrality value $d=1$. With the $k$ and $\lambda$ values set, the control limits $h_{i}$ are estimated for the control charts to obtain the same average run length. To standardise the analysis with other studies, the charts are calibrated to an $A R L_{0}$ of 200 , which is an intermediate value for the mean time, after which a false alarm may be triggered. Defining in-control thresholds for each chart using the same $A R L_{0}$ ensures an equivalent type I error for the tests under the null hypothesis of no change in the process.

When the calibration procedure is complete, we compare the chart $A R L_{1}$ values, i.e., the chart performance with a shift in the process. When the underlying process is actually out- of-control with a mean vector shift, a smaller $A R L_{1}$ value corresponds to better chart performance. Conversely, the chart may trigger a signal for a different variation source, indicating that the chart is not robust for different causes of variation. Thus, the $A R L_{1}$ computed for other sources of variation and different mean vector shifts can be viewed as a disadvantage or lack of robustness of the chart and treated as a scale of sensibility. The ARL empirical computation is described in the next section. All routines for the experiments were elaborated using the R environment (2008).

As previously stated, the Hotelling's $T$ control chart calibration is achieved using the process sample estimates or the known parameters to compute the thresholds by means of asymptotic distributions. An additional simple Hotelling's $T$ control chart calibration observes the false alarm rate and computes the probability of a signal, but we do not apply this procedure because the non-Shewhart control charts do not share this signal independence property. To compare the performance of non-Shewhart and Shewhart-type control chart, we choose the method described below in lieu of the traditional Markov Chain or integral equations approach for the MCUSUM and MEWMA threshold estimation.

\section{CONTROL CHART CALIBRATION BY LINEAR REGRESSION}

In this work, the control chart calibration is computed by specifying a sequence of approximate thresholds for each control chart and recording the resulting in-control average run length $\left(A R L_{0}\right)$. Then, a linear regression model in the form $\ln \left(\mathrm{ARL}_{0}\right)=a+b * h_{i}$ is fitted to estimate the target threshold for a predefined $\mathrm{ARL}_{0}=200$.
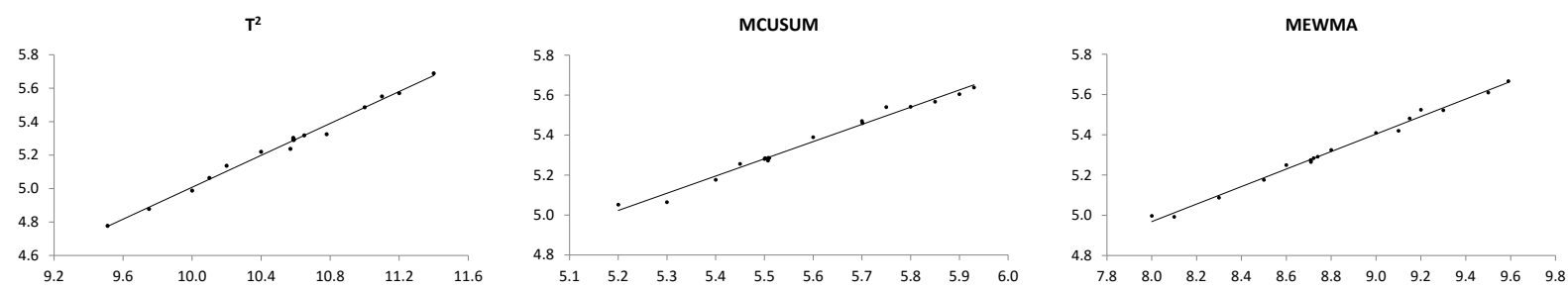

Figure 1. Linear regression adjustments for the three control charts based on known parameters

To access the $A R L_{0}$ estimation for each threshold value, $m$ is set equals 2,000 . This quantity of observations show

high probability (> 99.9\% for $\mathrm{ARL}_{0}=200$ ) of triggering a false alarm when the process is actually in-control. When observed, the position $N$ of the first alarm occurrence is recorded as the run length, and the mean value of $N$, computed from $B$ Monte Carlo simulations, is the $A R L_{0}$ for that threshold. In the experiments performed with known parameters and 20,000 Monte Carlo simulations, the maximum observed run length is 1,844 . To avoid missing values when the signal does not occur, $N$ is set to a maximum of 2,000. The experiments are performed with $B=2,000$ simulations to speed up the regression adjustment step, and $B=20,000$ simulations are performed with the estimated incontrol limit for the final $A R L_{0}$ and $A R L_{1}$ computation.

To illustrate the threshold sensitivity in the Hotelling's T control chart, Table 1 contains the asymptotic values for a Phase II control chart, based on the $F$ distribution, and respective standard errors obtained in 100,000 Monte Carlo simulations, where $m=100$ observations of the in-control 
process were simulated each step for parameter estimation.

The highest threshold $\left(h_{0.999}=15.13\right)$ corresponds to an $A R L_{0}$ value of 1,000 observations. Similarly, the confidence level of $99.5 \%\left(h_{0.995}=11.42\right)$ shows an average run length that is five times less (200.7) than the first one.

With the asymptotic values of Table 2 for the Hotelling's $\mathrm{T}$ chart and the reference values in the original papers for the MEWMA and MCUSUM charts, the linear regressions displayed in the Figure 1 are fitted with known parameters for all charts' calibrations. The minimum number of threshold values for the regression estimation is 10 , and the maximum is 18 . The values along the vertical axes are $A R L_{0}$ values in a logarithmic scale, and the values along the horizontal axes are the in-control limits.

Table 1. Confidence level, thresholds, $A R L_{0}$ and standard errors for a Phase II T control chart

\begin{tabular}{cccc}
\hline$(1-\alpha) 100 \%$ & $h_{1}$ & $\mathrm{ARL}_{0}$ & $\mathrm{SE}$ \\
\hline 99.9 & 15.13 & 1000.1 & 3.127 \\
99.5 & 11.42 & 200.7 & 0.625 \\
99.0 & 9.85 & 99.7 & 0.316 \\
95.0 & 6.30 & 19.9 & 0.063 \\
\hline
\end{tabular}

The parameter estimation is carried out with $m=$ 25, 50 and 100 for the number of Phase I samples of individual observations. The thresholds value sequences for estimating the regression model of each control chart are set to approximate the $A R L_{0}$ between 100 and 300. All fitted regressions, goodness-of-fit and estimated thresholds for a target $A R L_{0}=200$ are shown in Table 3.

Table 2. Asymptotic thresholds of Hotelling's T Phases I and II

\begin{tabular}{cccc}
\cline { 3 - 4 }$m$ & & Control chart type & \\
\hline & $X^{2}$-distribution & Beta-distribution & F-distribution \\
\hline 25 & - & 8.81 & 14.61 \\
50 & - & 9.69 & 12.34 \\
100 & - & 10.14 & 11.42 \\
$\left(\mathbf{M}_{0}, \boldsymbol{\Sigma}_{0}\right)$ & 10.60 & - & - \\
\hline
\end{tabular}

Table 3. Adjusted linear regression models with sample estimates and known parameters

\begin{tabular}{|c|c|c|c|c|}
\hline Control Chart & $m$ & Fitted model & $r^{2}$ & $\hat{h}_{i}\left(\mathrm{ARL}_{0}=200\right)$ \\
\hline \multirow{4}{*}{$\mathrm{T}$} & 25 & $\ln \left(A R L_{0}\right)=0.3710 * h_{1}+1.1206$ & 0.956 & 11.26 \\
\hline & 50 & $\ln \left(A R L_{0}\right)=0.4628 * h_{1}+0.2414$ & 0.971 & 10.93 \\
\hline & 100 & $\ln \left(A R L_{0}\right)=0.4954 * h_{1}+0.0463$ & 0.985 & 10.60 \\
\hline & $\left(\mathbf{M}_{0}, \mathbf{\Sigma}_{0}\right)$ & $\ln \left(A R L_{0}\right)=0.4773 * h_{1}+0.2351$ & 0.992 & 10.61 \\
\hline \multirow{4}{*}{ MCUSUM } & 25 & $\ln \left(A R L_{0}\right)=0.5138 * h_{2}+1.7467$ & 0.974 & 6.91 \\
\hline & 50 & $\ln \left(A R L_{0}\right)=0.6589 * h_{2}+1.1829$ & 0.993 & 6.25 \\
\hline & 100 & $\ln \left(A R L_{0}\right)=0.7343 * h_{2}+0.9318$ & 0.997 & 5.95 \\
\hline & $\left(\mathbf{M}_{0}, \boldsymbol{\Sigma}_{0}\right)$ & $\ln \left(A R L_{0}\right)=0.8611 * h_{2}+0.5451$ & 0.986 & 5.52 \\
\hline
\end{tabular}




\begin{tabular}{ccccc}
\hline & 25 & $\ln \left(A R L_{0}\right)=0.2811 * h_{3}+2.0399$ & 0.963 & 11.59 \\
MEWMA & 50 & $\ln \left(A R L_{0}\right)=0.3628 * h_{3}+1.5648$ & 0.974 & 10.29 \\
& 100 & $\ln \left(A R L_{0}\right)=0.3892 * h_{3}+1.5511$ & 0.994 & 9.63 \\
& $\left(\mathbf{M}_{0}, \mathbf{\Sigma}_{0}\right)$ & $\ln \left(A R L_{0}\right)=0.4348 * h_{3}+1.4904$ & 0.993 & 8.76 \\
\hline
\end{tabular}

We first compare the estimated thresholds with the asymptotic values of Hotelling's T control charts presented in Table 2. In Table 3, we notice that the linear regression approach shows a tendency of larger thresholds for smaller sample sizes, as expected, and the estimate for known parameters (10.61) is very close to the asymptotic value (10.60). Second, for sample estimates, the regression adjusted thresholds are close to the asymptotic average between the two distributions. For $m=25$, the asymptotic threshold average is 11.71, while the regression estimate is 11.26. For $m=50$ and 100, the asymptotic threshold averages are 11.02 and 10.78, respectively, while the regression estimates are 10.93 and 10.60 . Thus, the linear regression approach is effective for calibrating Hotelling's $T$ control chart.
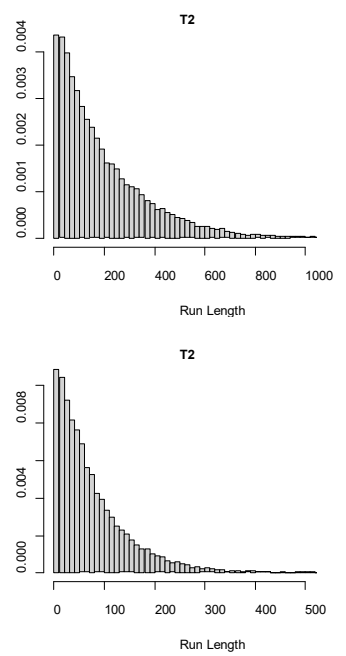

T2

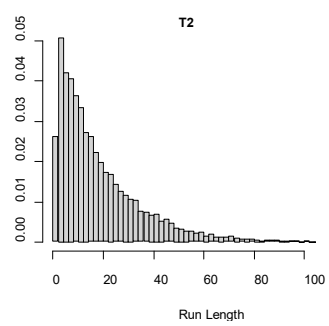

T2

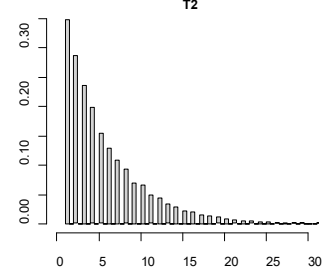

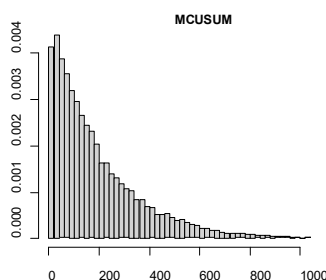

a) $\stackrel{\text { Run Length }}{=}=0$

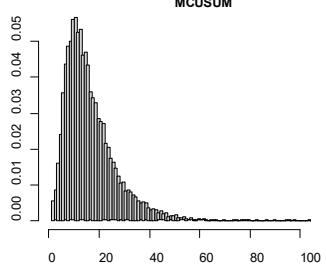

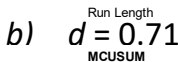

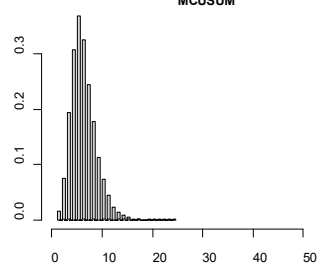

c) $d \stackrel{\text { Run Length }}{=} 1.41$

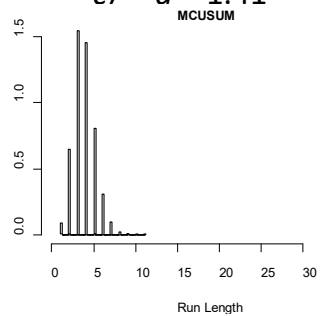

d) $d=2.12$

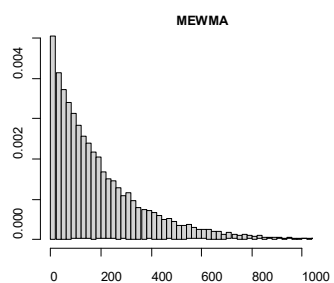

Run Length
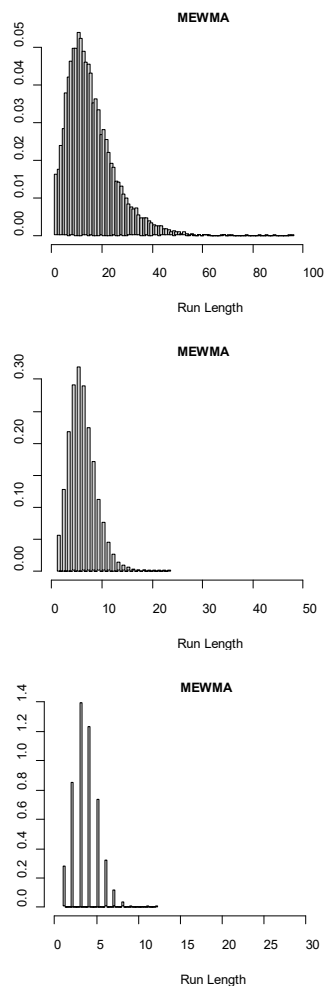

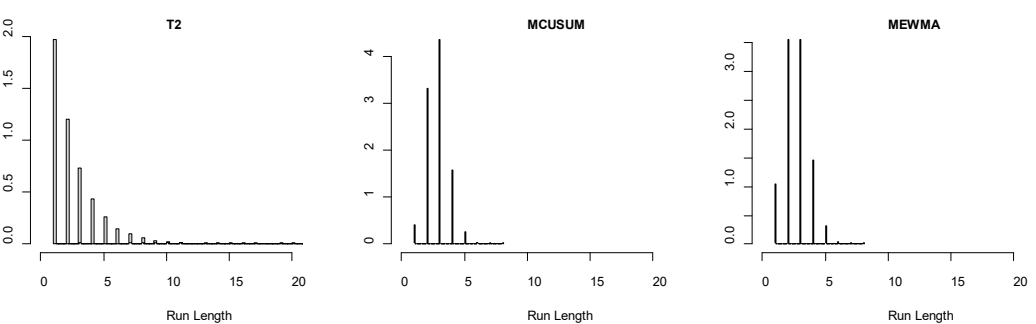

T2

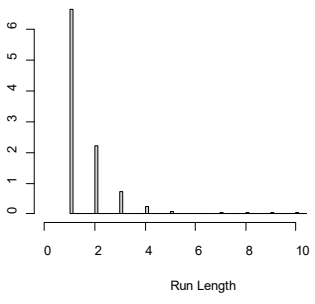

e) $d=2.82$
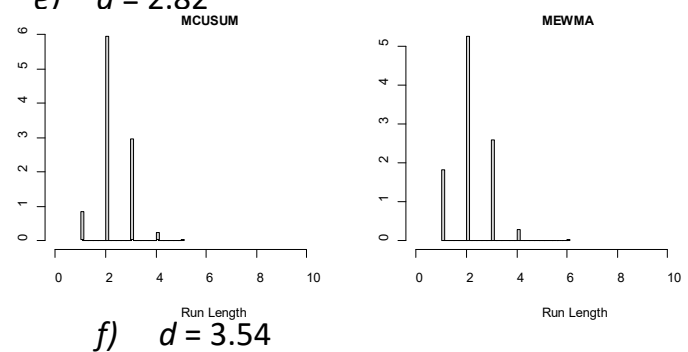

Figure 2. Empirical in- and out-of-control run length distributions

\section{IN-CONTROL AND OUT-OF-CONTROL RUN LENGTH DISTRIBUTIONS}

To compare the run length distributions calibrated with known parameters, Figure 2 presents a sequence of density histograms based on the resulting run length values for 20,000 Monte Carlo simulations. Figure 2-(a) shows the run length distributions for the three control charts and the mean points of each distribution are the $A R L_{0}$ values of the last group in Table 4, which is based on the known parameters $\left(\mathbf{M}_{0}, \boldsymbol{\Sigma}_{0}\right)$

Some large observations are not displayed in the histograms because the horizontal axes were set to not lose scale definition. The maximum value in the mean vector shifts of Figure (b) and (c) was set differently for the Hotelling's $T$ control chart to improve visualisation. When the shift is large as in Figure 2-(f), the Hotelling's $T$ is clearly the most favourable to change detection. The run length distribution of the MEWMA control chart as seen Figure 2 (e) and (f) confirm that this control chart with $\lambda=0.1$ tends to perform better than the MCUSUM with $k=0.5$. This result is also confirmed by the $A R L_{1}$ values produced in the experiments with known parameters of Table 4.

Simulating long runs for the $(2,000)$ is not necessary for defining the out-of-control run length distributions. For the $A R L_{1}$ estimation, the total number of simulated observations utilised for each mean vector shift was 1,500, 1,000, 500, 200 and 100 , which considerably reduced the computing time costs.

\section{PERFORMANCE COMPARISON FOR MEAN VECTOR SHIFTS}

Excluding the specific mean vector shifts to test the MCUSUM method, Crosier (1988) evaluates $d$ unitary increments in the 0 to 5 range for several dimensions. Lowry and Montgomery (1995) in the MEWMA method evaluate shifts through $d$ increments in the 0 to 3 range. The present work simulates changes in equal increments for both dimensions, and the centre of the process is shifted diagonally from $(0.0,0.0)$ to $(2.5,2.5)$ by increments of 0.5 in both dimensions, representing $d$ values in the 0 to 3.54 range. The probability inside the area defined by three standard deviations in a non-correlated process is $\mathbf{0 . 9 9 5}$.

Figure 3 illustrates the proposed mean vector shifts for correlated and non-correlated processes. Only the scheme in Figure 3-(a) is calibrated with estimated and known parameters. The correlated variable schemes (b) and (c) of Figure 3 are compared only for the case of known parameters. The experienced reader should argue why to do comparisons with correlated variables since the charts performance does not depends on the shifts direction. For that reasoning, the authors intend to emphasize that a shift of magnitude $d$ is different when it occurs in the process directions of major and minor variability.

For non-correlated processes shown in Figure 3-(a), the in-control limits are delimited with known parameters and sample estimates of three different sizes in Phase I. Table 4 presents the resulting in- and out-of-control ARLs. Although the standard error (SE) of the estimate is relatively large for the $A R L_{0}$ due to the regular number of Monte Carlo simulations $(B=20,000)$, the $A R L_{1}$ values show little variation. 


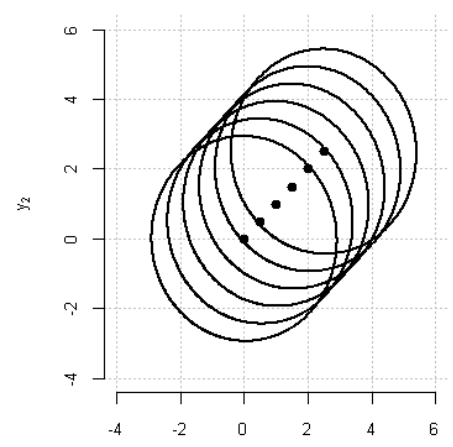

(a) $\stackrel{y_{1}}{\rho}=0$

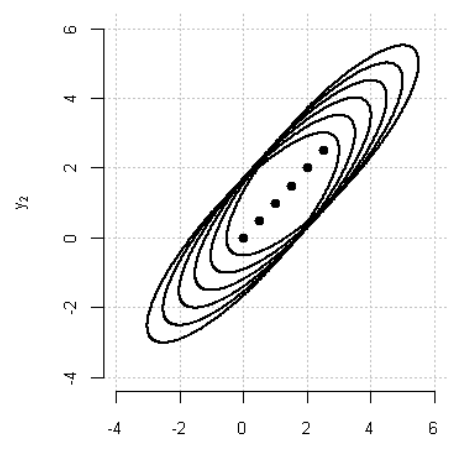

(b) $\rho=+0.85$

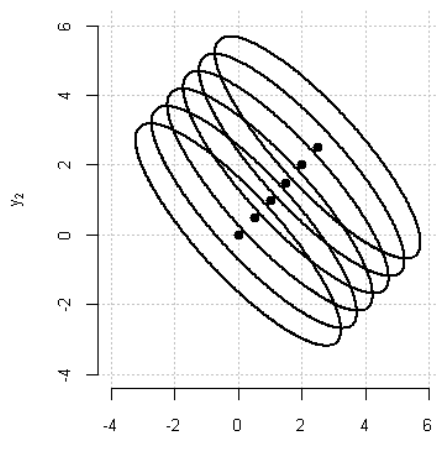

(c) $\rho=-0.85$

Figure 3. Diagram of the proposed mean vector shifts for non-correlated and correlated processes

The main observed effect due to the utilisation of small sample sizes to train the control chart in Table 4 is an increasing delay in the change detection as the sample size decreases. The MCUSUM and MEWMA charts perform very similarly for all shifts, with faster performance than the Hotelling's T chart for the shifts $(2.0,2.0)$ that are situated around the target non-centrality value $(d=1)$. To this point, the inertia effect begins to delay the change detection for the non-Shewhart charts, and the shift for the point $(2.5$, 2.5) is more likely to be detected with the Shewhart-type control chart.

The experiments with modified correlation structures shown in Figure 3 parts (b) and (c) determine the changes only in the first quadrant, thus allowing changes to represent both large and small shifts respective to the noncentrality parameter. Figure 4 demonstrates how much those different structures allow the same shift in the mean vector to represent a relatively different shift in the noncentrality parameter. Compared with the non-correlated process represented by the solid line, the mean vector shifts measured by the non-centrality parameter are smaller in the positively correlated process (gray line) and larger in the negatively correlated process (dashed line).

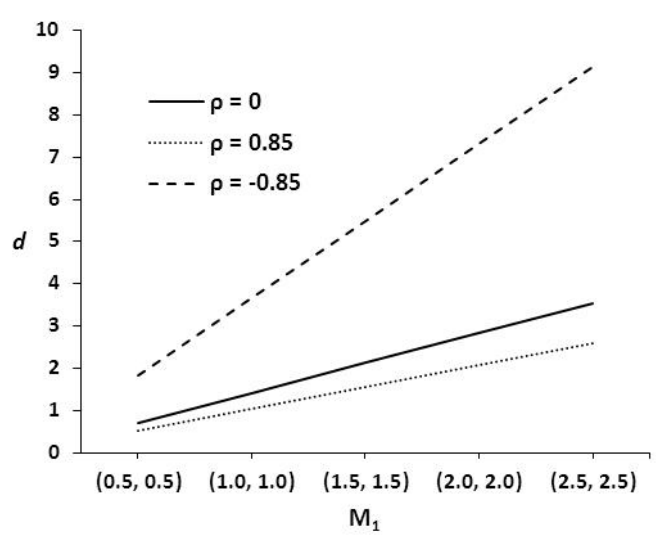

Figure 4. Shift size with respect to the noncentrality parameter in the correlated and non-correlated processes

Throughout the rest of the experiments, the in-control limits are obtained by calibrating the control chart with the process known parameters. The results for ARL comparison in detecting the process shift schemes of Figure 3 are shown in Table 5 and represented in $d$ units in Figure 5. Although the MCUSUM and MEWMA charts have equivalent performance in the three situations, the performance of the Hotelling's T chart exceeds the others after the first shift in Figure 5 case (c), when the shift occurs in the minor axis of the negatively correlated process. This result does not contradict the finding that the Hotelling's T chart is a better monitor of large shifts. An observation of the point where the charts intersect indicates that the relative magnitude of the changes in all three cases is the same when measured using the noncentrality parameter $(d \cong 2.6)$. 


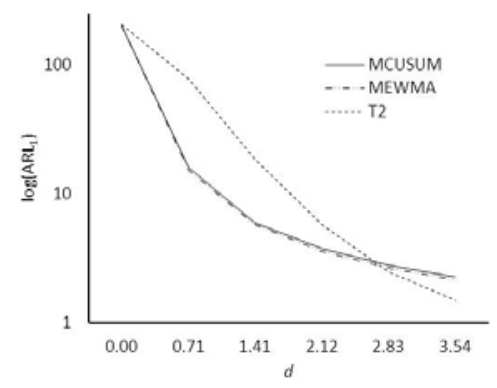

(a) $\rho=0$

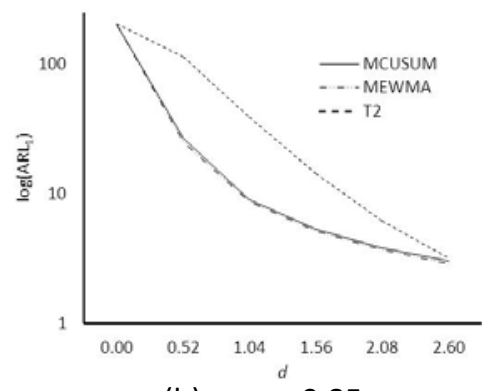

(b) $\rho=+0.85$

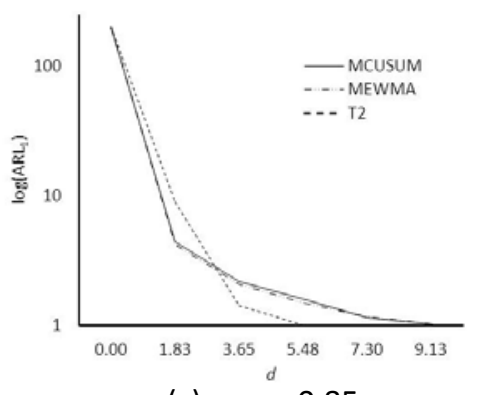

(c) $\rho=-0.85$

Figure 5. The control charts' performance for the three simulated processes and mean vector shifts as non-centrality values

This situation occurs because a large distance between the processes tends to result in inertia in the MCUSUM and MEWMA methods (Lowry et al., 1992), delay change detection and make the Hotelling's $T$ chart more efficient in the third shifting scheme. We observe that larger shifts often occur in the direction of larger variability. A more realistic suggestion in this case is to observe relatively less movement in the direction of minor variability. The large values in the direction of lower variability are usually related to typing errors in the data acquisition procedure.
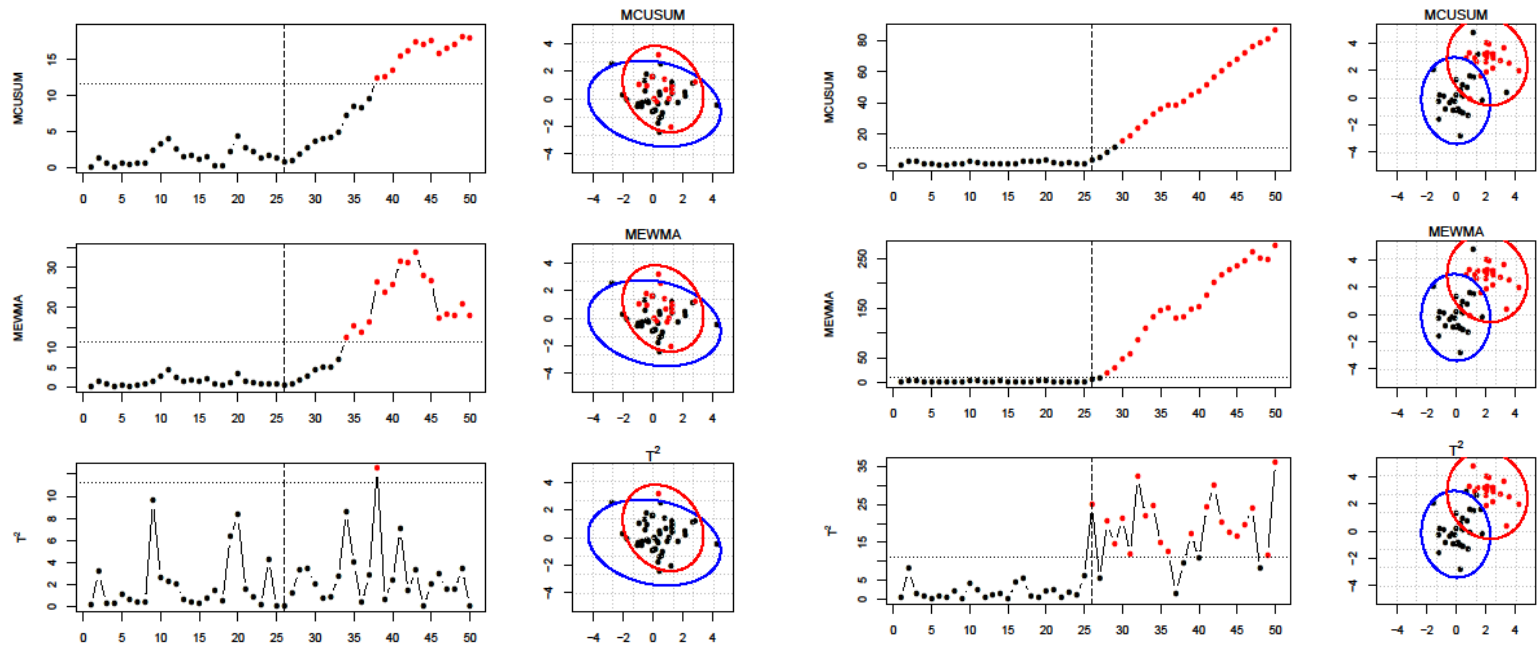

(a) $d=1.41 ; \mathbf{M}_{1}=(1.0,1.0)$

(b) $d=3.54 ; \mathbf{M}_{1}=(2.5,2.5)$

Figure 6. Control chart patterns for moderate and large mean vector shifts 

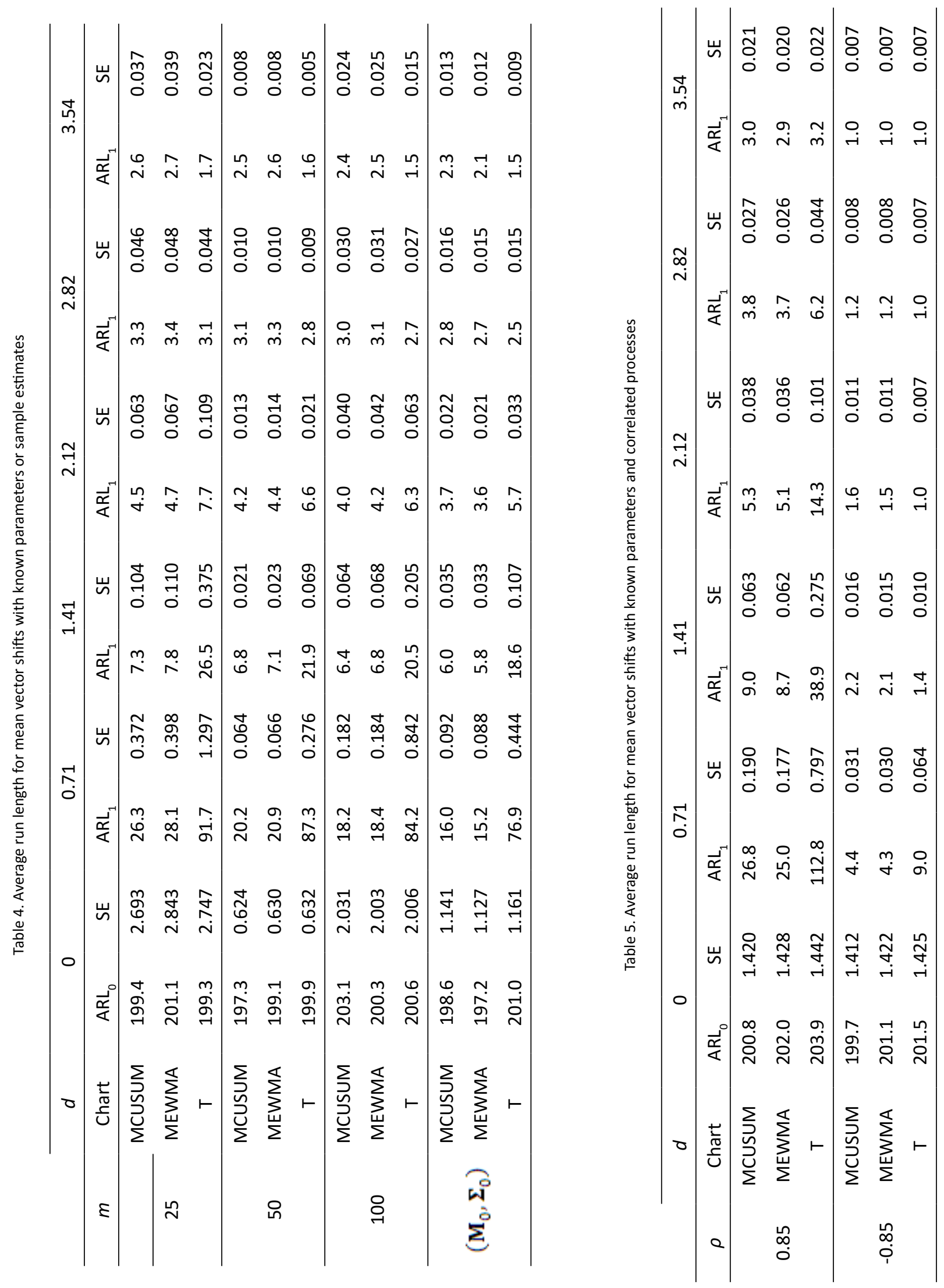
Figure 6 illustrates three simulated runs for mean vector shifts in the process. The changes in the processes are moderate and large shifts. The inertia effect can be viewed in part (b) with the non-Shewhart control charts. As the Hotelling's T chart triggers a signal at the very first out-ofcontrol observation, the MCUSUM and MEWMA charts are affected by a delay in triggering the signal.

\section{THE INFLUENCE OF INCREASING VARIANCES}

If the variances of the underlying process changes from $\left(\sigma_{1}^{2}, \sigma_{2}^{2}\right)$ to $\left(\sigma_{1}^{2}+\Delta, \sigma_{2}^{2}+\Delta\right)=\left(\sigma_{1}^{2}, \sigma_{2}^{2}\right)^{*}$, control chart may be affected because this overdispersion increases the individual observation distances with respect to the center of the in-control process. The results in Table 6 reflect the effect of process variance increases, which demonstrates a larger influence on the Hotelling's T control chart.

The patterns that result from simultaneously altering the mean vector and covariance matrix generate a greater tendency for signal growth than the pattern shown in part (a) of Figure 7. When the mean vector changes, the subsequent process changes stemming from the modification of the covariance matrix greatly influence the charts' performance. Whereas the MCUSUM and MEWMA methods consider the entire process to be out-of-control, beginning with the characterisation of a new centre of gravity, the Hotelling's T chart discriminates all of the observations that exceed the coverage area, as defined by the in-control process.

The difference in the charts' performance can be observed by comparing Tables 6 and 7. Table 7 shows the influence of variance increasing over the mean vector shifts. This experiment performs only a slight increase in the variances, which changes from $\left(\sigma_{1}^{2}, \sigma_{2}^{2}\right)=(1.0,1.0)$ to $\left(\sigma_{1}^{2}, \sigma_{2}^{2}\right)^{*}=(1.5,1.5)$. The combined effect of the increased variances and mean vector shifts is more evident for the Hotelling's T control chart, where the reductions in the $\mathrm{ARL}_{1}$ are $73 \%, 52 \%, 30 \%, 11 \%$ and $0 \%$. The MCUSUM and MEWMA control charts demonstrate a significant difference in the $A R L_{1}$ for the first shift only, which are reduced by $16 \%$ and $15 \%$, respectively. The $A R L_{1}$ reduction for the nonShewhart charts in the second shift is only $3 \%$ and is not significant for bigger shifts.

Table 6 . The influence of increasing the process variances on the average run length

\begin{tabular}{ccccccccc}
\hline$\left(\sigma_{1}^{2}, \sigma_{2}^{2}\right)^{*}$ & \multicolumn{2}{c}{$(1.0,1.0)$} & \multicolumn{2}{c}{$(1.5,1.5)$} & \multicolumn{2}{c}{$(2.0,2.0)$} & \multicolumn{2}{c}{$(2.5,2.5)$} \\
\hline Control chart & $\mathrm{ARL}_{0}$ & $\mathrm{SE}$ & $\mathrm{ARL}$ & $\mathrm{SE}$ & $\mathrm{ARL}_{1}$ & $\mathrm{SE}$ & $\mathrm{ARL}_{1}$ & $\mathrm{SE}$ \\
\hline MCUSUM & 198.1 & 1.401 & 52.3 & 0.370 & 26.8 & 0.190 & 17.9 & 0.126 \\
MEWMA & 202.8 & 1.434 & 56.3 & 0.398 & 29.0 & 0.205 & 18.9 & 0.134 \\
$\mathrm{~T}$ & 202.3 & 1.431 & 35.0 & 0.247 & 14.2 & 0.100 & 8.4 & 0.060 \\
\hline
\end{tabular}
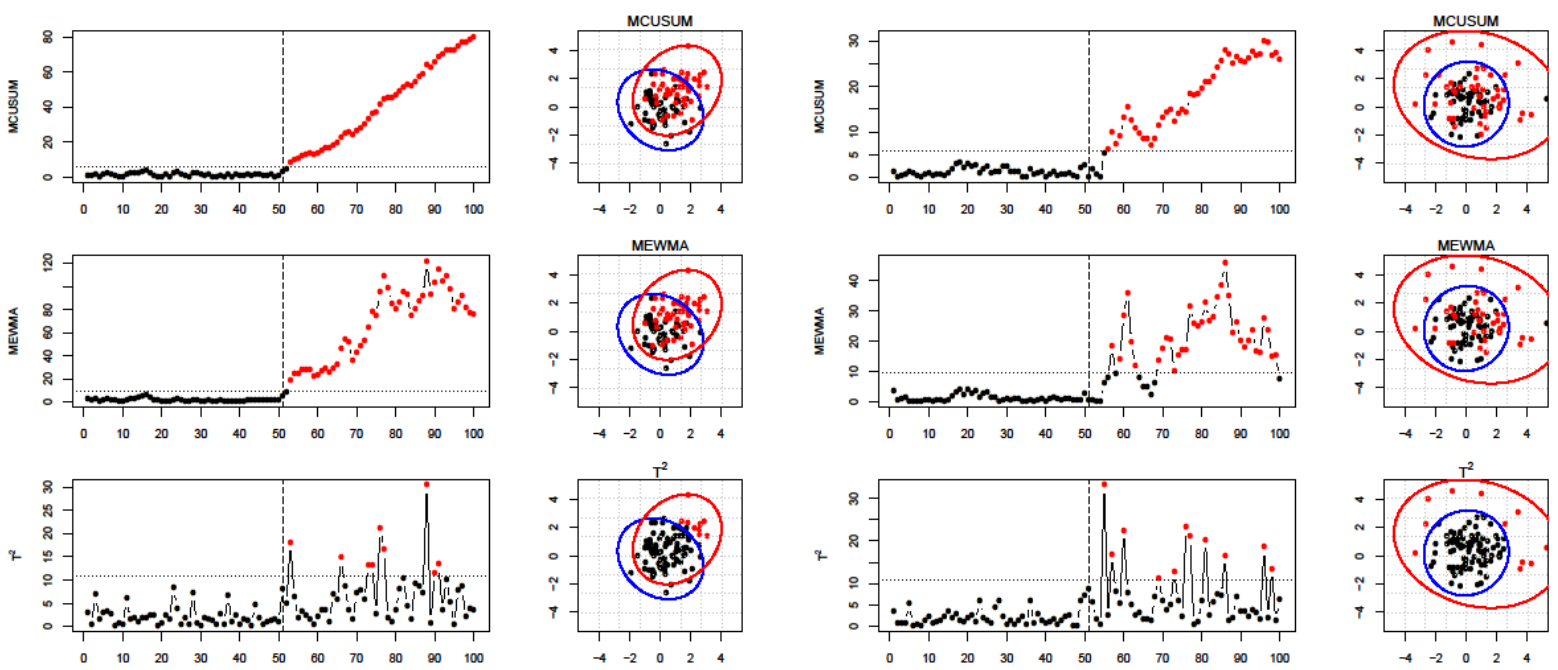

(b)

$$
\mathbf{M}_{1}=(1.0,1.0), \mathbf{\Sigma}_{1}=\left[\begin{array}{ll}
2 & 0 \\
0 & 2
\end{array}\right]
$$

(a)

$$
\mathbf{M}_{1}=(1.0,1.0), \mathbf{\Sigma}_{1}=\left[\begin{array}{ll}
1 & 0 \\
0 & 1
\end{array}\right]
$$


Table 7. The ARL influence of simultaneously increasing the variances and shifting the mean vector

\begin{tabular}{ccccccccccccccc}
\hline$\left(\sigma_{1}^{2}, \sigma_{2}^{2}\right)^{*}$ & \multicolumn{1}{c}{$(1.0,1.0)$} & \multicolumn{1}{c}{0} & \multicolumn{1}{c}{$(1.5,1.5)$} \\
\hline$d$ & \multicolumn{2}{c}{0} & \multicolumn{2}{c}{0.71} & \multicolumn{2}{c}{1.41} & \multicolumn{2}{c}{2.12} & \multicolumn{2}{c}{2.82} & \multicolumn{2}{c}{3.54} \\
\hline Control chart & $\mathrm{ARL}_{0}$ & $\mathrm{SE}$ & $\mathrm{ARL}$ & $\mathrm{SE}$ & $\mathrm{ARL}$ & $\mathrm{SE}$ & $\mathrm{ARL}$ & $\mathrm{SE}$ & $\mathrm{ARL}_{1}$ & $\mathrm{SE}$ & $\mathrm{ARL}_{1}$ & $\mathrm{SE}$ \\
\hline MCUSUM & 198.5 & 1.404 & 13.5 & 0.095 & 5.8 & 0.041 & 3.7 & 0.026 & 2.8 & 0.020 & 2.3 & 0.016 \\
MEWMA & 198.2 & 1.402 & 13.0 & 0.092 & 5.6 & 0.040 & 3.6 & 0.025 & 2.7 & 0.019 & 2.2 & 0.015 \\
$\mathrm{~T}$ & 200.8 & 1.420 & 21.3 & 0.151 & 8.9 & 0.063 & 4.1 & 0.029 & 2.2 & 0.016 & 1.5 & 0.011 \\
\hline
\end{tabular}

\section{THE INFLUENCE OF AUTOCORRELATION}

To verify the control charts' performance when the process is perturbed with autocorrelation, the out-ofcontrol processes are simulated based on a first-order autoregressive model, VAR(1), on a scale of increasing intensity. The autocorrelations in both dimensions are $\Phi=\left(\varphi_{1}, \varphi_{2}\right)=(0.0,0.0)$ for the in-control process and $\boldsymbol{\Phi}=\left(\varphi_{1}+\Delta, \varphi_{2}+\Delta\right)=(\Delta, \Delta)$ for the out-of-control process. The autocorrelation levels are $\Delta=\{0.1,0.2,0.3,0.4,0.5,0.6,0.7,0.8,0.9\}$. The degree of influence on the processes is indirectly evaluated by comparing the $A R L_{1}$ values produced in the experiments described in Tables 8 and 9 to the values presented in Table 4 , where only the mean vector was modified.

The experiment in Table 8 demonstrates that pure autocorrelation in the out-of-control process results in small mean vector shifts, being less noticed by the Hotelling's $T$ chart. The experiment in Table 9 inserts low autocorrelation levels in the process and simultaneously shifts the mean vector. The combined effect of mean vector shifts and autocorrelation shows a reduction in the $A R L_{1}$ primarily for small shifts with all control charts and an acceleration of the change detection as the autocorrelation level increases. Further, the increase in the autocorrelation with mean vector shifts demonstrates a bigger influence on the Hotelling's T chart. Comparing with simply shifting the mean vector to $\mathbf{M}_{1}=(0.5,0.5)$, the experiments utilising $\Phi=(0.1,0.1)$ and $\mathbf{M}_{1}=(0.5,0.5)$ accelerate the Hotelling's T chart to identify the first two smaller shifts by $17.7 \%$ and $23 \%$, while the MCUSUM and MEWMA charts are accelerated by $18 \%$ and $16.3 \%$, respectively. When $\Phi=(0.2,0.2)$, the acceleration in change detection are also more evident for the small shifts in the mean vector.

Table 8. The ARL influence of purely increasing autocorrelation levels

\begin{tabular}{|c|c|c|c|c|c|c|c|c|c|c|}
\hline \multirow{2}{*}{$\begin{array}{c}\Phi \\
\text { C. Chart } \\
\end{array}$} & \multicolumn{2}{|c|}{$(0.0,0.0)$} & \multicolumn{2}{|c|}{$(0.1,0.1)$} & \multicolumn{2}{|c|}{$(0.2,0.2)$} & \multicolumn{2}{|c|}{$(0.3,0.3)$} & \multicolumn{2}{|c|}{$(0.4,0.4)$} \\
\hline & $\mathrm{ARL}_{0}$ & SE & $\mathrm{ARL}_{1}$ & SE & $\mathrm{ARL}_{1}$ & SE & $\mathrm{ARL}_{1}$ & SE & $\mathrm{ARL}_{1}$ & SE \\
\hline MCUSUM & 198.5 & 1.404 & 105.3 & 0.744 & 60.1 & 0.425 & 36.1 & 0.255 & 23.9 & 0.169 \\
\hline MEWMA & 198.2 & 1.402 & 111.1 & 0.785 & 64.7 & 0.457 & 39.8 & 0.282 & 25.9 & 0.183 \\
\hline$T$ & 200.8 & 1.420 & 194.0 & 1.372 & 163.6 & 1.157 & 130.4 & 0.922 & 92.2 & 0.652 \\
\hline$\Phi$ & \multicolumn{2}{|c|}{$(0.5,0.5)$} & \multicolumn{2}{|c|}{$(0.6,0.6)$} & \multicolumn{2}{|c|}{$(0.7,0.7)$} & \multicolumn{2}{|c|}{$(0.8,0.8)$} & \multicolumn{2}{|c|}{$(0.9,0.9)$} \\
\hline C. Chart & $\mathrm{ARL}_{1}$ & SE & $\mathrm{ARL}_{1}$ & SE & $\mathrm{ARL}_{1}$ & SE & $\mathrm{ARL}_{1}$ & SE & $\mathrm{ARL}_{1}$ & SE \\
\hline MCUSUM & 16.4 & 0.116 & 11.7 & 0.083 & 8.5 & 0.060 & 6.2 & 0.044 & 4.6 & 0.032 \\
\hline MEWMA & 17.4 & 0.123 & 12.2 & 0.086 & 8.7 & 0.061 & 6.2 & 0.044 & 4.5 & 0.032 \\
\hline$T$ & 60.5 & 0.428 & 37.7 & 0.266 & 21.8 & 0.154 & 12.8 & 0.090 & 7.1 & 0.051 \\
\hline
\end{tabular}

To visualise the effect of pure autocorrelation in the out-of-control process, Figure 8 illustrates the action pattern of the control charts when $\boldsymbol{\Phi}=(0.8,0.8)$. The non-Shewhart control charts are more likely to detect the change of an oscillatory movement in the process, which is characteristic of positive autocorrelation. Observing the three-dimensional scatter plots of Figure 8 , this variation source initially performs a small shift in the process mean vector.

In all cases, the distances measured using the noncentrality parameter suggest that the effects resulting from purely autocorrelated processes favour detection by the MCUSUM and MEWMA charts. The individual observation distances do not represent significant shifts relative to the in- 
control process. In situations when a shift in the mean vector occurs with a regular autocorrelation level, the Hotelling's T chart can be more effective to change detection.

The MCUSUM and MEWMA charts are generally considered effective for detecting shifts that are caused by the occurrence of purely positive autocorrelation at regular and high levels; for negative autocorrelation, the Hotelling's $T$ chart is most suitable to perceive the changes as outliers in the process.

Table 9. The ARL influence of simultaneously increasing autocorrelations and shifting the mean vector

\begin{tabular}{|c|c|c|c|c|c|c|c|c|c|c|c|c|}
\hline \multirow{3}{*}{$\begin{array}{c}\boldsymbol{\Phi} \\
d \\
\text { C. Chart }\end{array}$} & \multicolumn{6}{|c|}{$(0.0,0.0)$} & \multicolumn{6}{|c|}{$(0.5,0.5)$} \\
\hline & \multicolumn{2}{|c|}{0} & \multicolumn{2}{|c|}{0.71} & \multicolumn{2}{|c|}{1.41} & \multicolumn{2}{|c|}{2.12} & \multicolumn{2}{|c|}{2.82} & \multicolumn{2}{|c|}{3.54} \\
\hline & $\mathrm{ARL}_{0}$ & SE & $\mathrm{ARL}_{1}$ & SE & $\mathrm{ARL}_{1}$ & SE & $\mathrm{ARL}_{1}$ & SE & $\mathrm{ARL}_{1}$ & SE & $\mathrm{ARL}_{1}$ & SE \\
\hline MCUSUM & 201.0 & 1.421 & 13.2 & 0.093 & 5.3 & 0.037 & 3.4 & 0.024 & 2.5 & 0.018 & 2.1 & 0.015 \\
\hline MEWMA & 201.1 & 1.422 & 12.8 & 0.090 & 5.1 & 0.036 & 3.2 & 0.023 & 2.4 & 0.017 & 2.0 & 0.014 \\
\hline $\mathrm{T}$ & 203.1 & 1.457 & 64.2 & 0.454 & 14.2 & 0.100 & 4.4 & 0.031 & 2.0 & 0.014 & 1.3 & 0.009 \\
\hline$\Phi$ & \multicolumn{6}{|c|}{$(0.0,0.0)$} & \multicolumn{6}{|c|}{$(0.5,0.5)$} \\
\hline$d$ & \multicolumn{2}{|c|}{0} & \multicolumn{2}{|c|}{0.71} & \multicolumn{2}{|c|}{1.41} & \multicolumn{2}{|c|}{2.12} & \multicolumn{2}{|c|}{2.82} & \multicolumn{2}{|c|}{3.54} \\
\hline C. Chart & $\mathrm{ARL}_{0}$ & SE & $\mathrm{ARL}_{1}$ & SE & $\mathrm{ARL}_{1}$ & SE & $\mathrm{ARL}_{1}$ & SE & $\mathrm{ARL}_{1}$ & SE & $\mathrm{ARL}_{1}$ & SE \\
\hline MCUSUM & 201.0 & 1.421 & 10.8 & 0.077 & 4.6 & 0.033 & 3.0 & 0.021 & 2.8 & 0.020 & 2.3 & 0.016 \\
\hline MEWMA & 201.1 & 1.422 & 10.6 & 0.075 & 4.5 & 0.032 & 2.9 & 0.020 & 2.7 & 0.019 & 2.2 & 0.015 \\
\hline $\mathrm{T}$ & 202.1 & 1.457 & 48.4 & 0.342 & 10.2 & 0.072 & 3.2 & 0.023 & 2.2 & 0.016 & 1.5 & 0.011 \\
\hline
\end{tabular}

Figure 9 shows the negative autocorrelated out-ofcontrol process pattern results, which are only detected using the Hotelling's T chart, especially if the magnitude of the autocorrelation is large and the outliers appear beyond the in-control region. Conversely, the MCUSUM and MEWMA statistics result in no sensitivity to the negative autocorrelated process because the alternating individual observations cancel each other.

\section{DISCUSSION}

The present study investigates the behavioural patterns and performance of the control charts widely applied in SPC to monitor the mean vector, i.e., the MCUSUM, MEWMA and Hotelling's $T$ charts, with individual observations and combined sources of variability. The performance comparison of the charts with controlled simulated changes is carried out by the estimated ARLs. A procedure for the confidence ellipse estimation based on principal component analysis is briefly described as a descriptive tool for visualisation purposes.

As a unifying approach to estimate the in-control limits of Shewhart- and non-Shewhart-type control charts, the linear regressions of the thresholds as a function of the average run length are adjusted for known parameters and sample estimates of different sizes. The estimated thresholds for the three selected control charts agree with the values described in the literature. As noted above, an estimation of the control charts thresholds with a small number of individual Phase I observations increases the in-control limits and delays change detection for all studied control charts.

Different scenarios for the correlated process are established to compare the mean vector shifts in the directions of larger and smaller variations. These experiments demonstrate how the performance of selected control charts is dependent only on the shift magnitude, as measured by the noncentrality parameter. 

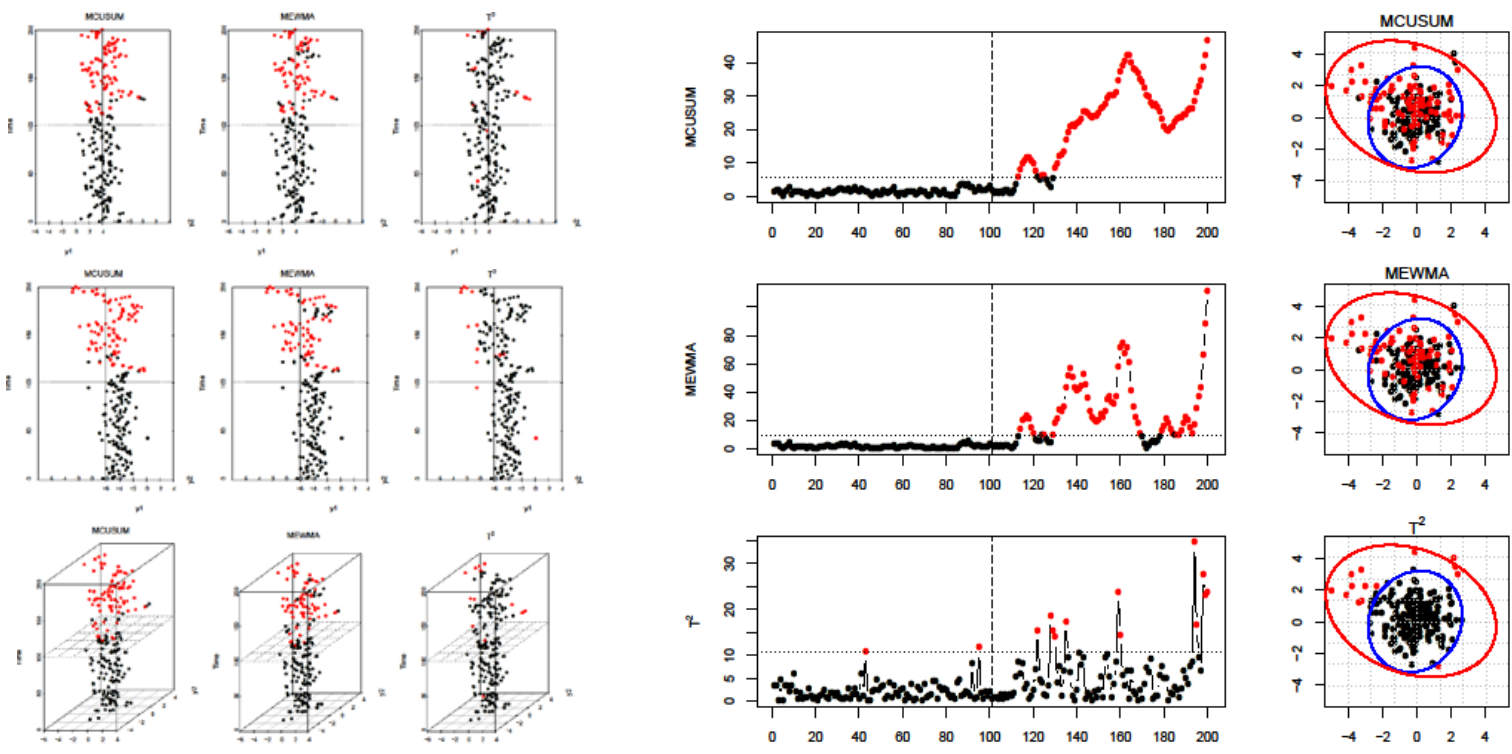

(a)
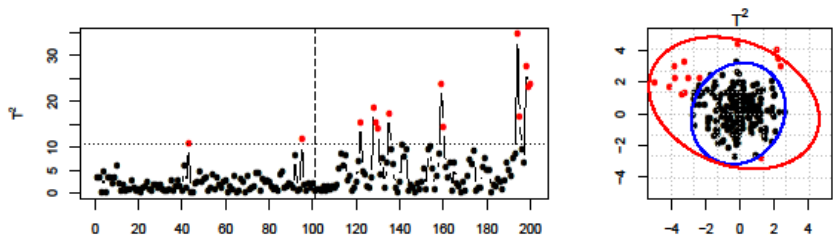

(b)

Figure 8. Three-dimensional scatter plots (a) and control charts with confidence ellipses (b) for a purely autocorrelated out-of-control process

$$
\Phi=(0.8,0.8)
$$
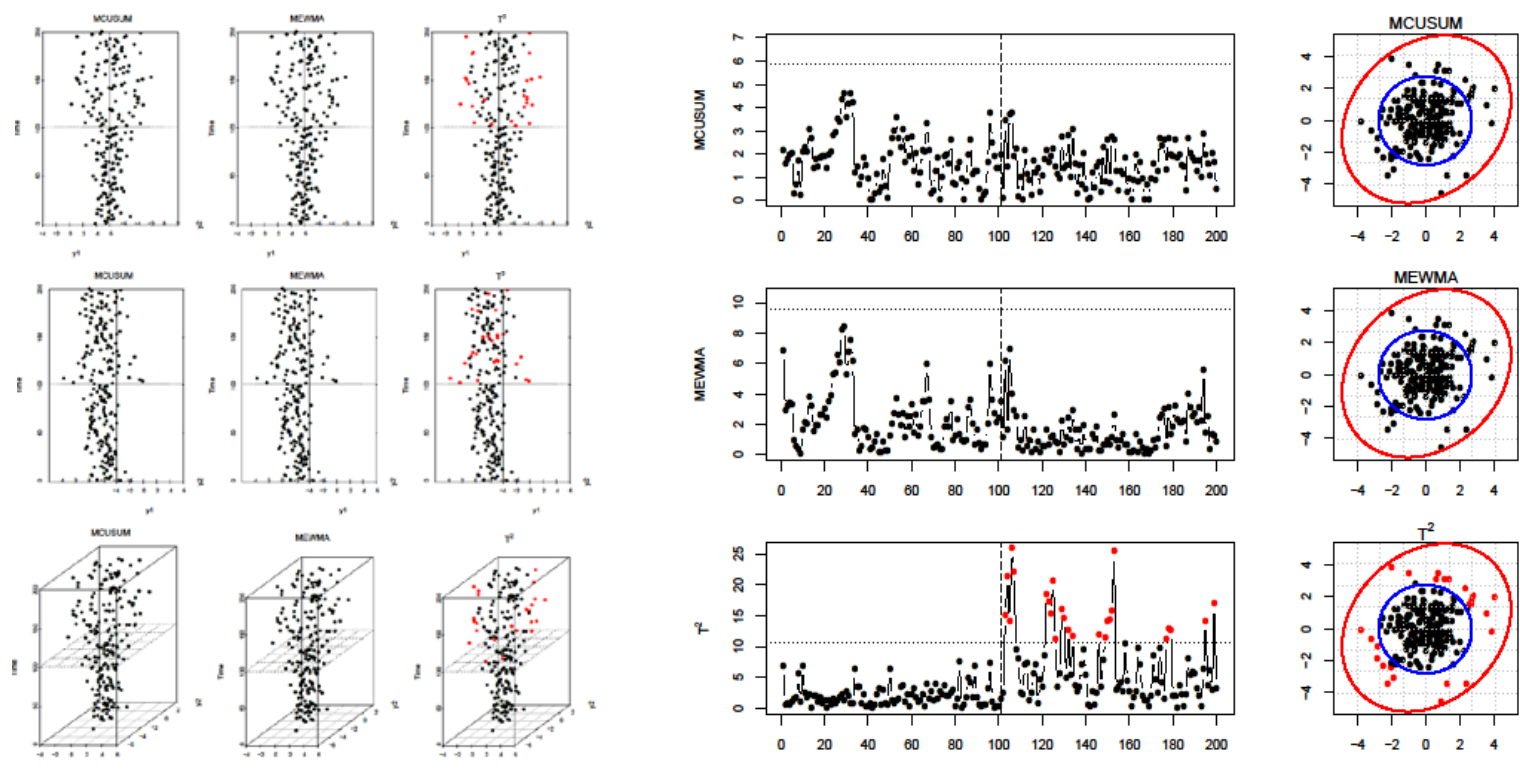

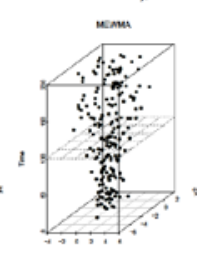

(a)

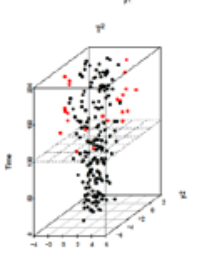

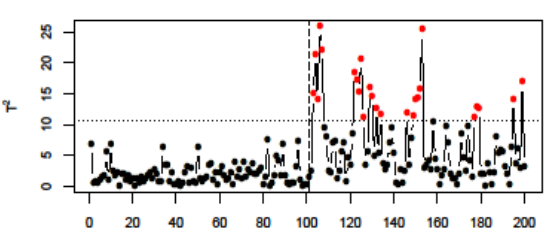

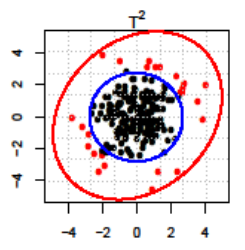

(b)

Figure 9. Three-dimensional scatter plots (a) and control charts with confidence ellipses (b) for the negative autocorrelated process

$$
\Phi=(-0.8,-0.8)
$$

The estimated in-control limits for known parameters are applied to study the effects of combining mean vector shifts and increasing variances or serial autocorrelations in the out-of-control process. We demonstrate that the resulting effect on the charts' performance following an increase only in the variances in the out-of-control process is more evident in the Hotelling's T chart, even for relatively small variance increments. The combined effect of jointly shifting the mean vector and increasing the variances in low levels makes the Hotelling's T control chart as competitive as the non-Shewhart charts, even for moderate shift sizes in the mean vector because the influence of increasing the process dispersion additionally increases the individual noncentrality values.

The effect of serial autocorrelation simulated in the outof-control process follows a first-order autoregressive model at different intensity levels. Among all proposed increments 
Brazilian Journal of Operations \& Production Management

Volume 12, Número 2, 2015, pp. 196-212

DOI: 10.14488/BJOPM.2015.v12.n2.a2

in the autocorrelation, the experiments in this work showed that the effects of purely autocorrelated processes can be identified as small shifts in the mean vector, with correspondingly better change detection by the MCUSUM and MEWMA charts. When combined with the mean vector shifts, low levels of positive autocorrelation demonstrate that the Hotelling's T control chart is as competitive as or better than the non-Shewhart charts for moderate and large shifts in the mean vector.

The control charts' performance in the presence of negative autocorrelation shows that only the Hotelling's $T$ chart recognises the effects as outliers due to their continuous process dispersion. Whereas the MCUSUM method voids the information about the shift when the observations produce statistics with alternating signs in the cumulative sums, the MEWMA chart becomes ineffective by weighting the current value with the historical average, which is also affected by continuously alternating observations.

The non-centrality parameter is widely applied in single-hypothesis problems to control the mean vectors in multivariate processes because the analysis of all possible alternative distributions prior to decision-making is impossible. This technique works well when the observation size is small, e.g., 1 or 2 . When $p$ increases, however, mapping the $p$-dimensional space for a one-dimensional distance may destroy valuable information for the correct classifications that exist in the original spaces, if the direction of the shift is unknown a priori. A careful search of the conditional discriminant functions that determine an ideal intersection between the processes can reveal an optimal procedure.

As previously noted, the literature on statistical pattern recognition proposes several dissimilarity measures to improve class separability. Measures specifically delineated for multivariate normal distributions, such as appropriate linear transformations of the Mahalanobis distance, show superior performance in ARL when compared with the charts in the present study, on which topic work will soon be published.

\section{ACKNOWLEDGEMENTS}

This research is supported by CAPES (Conselho de Aperfeiçoamento de Pessoal de Nível Superior, by REUNI (Programa de Apoio a Planos de Reestruturação e Expansão das Universidades Federais), by PRPq-UFMG (Pró-Reitoria de Pesquisa da Universidade Federal de Minas Gerais), and by PRRH-UFSM (Pró-Reitoria de Recursos Humanos da Universidade Federal de Santa Maria.

\section{REFERENCES}

Mahalanobis, P.C. (1936) On the Generalised Distance in Statistics. Proceedings of the National Institute of Sciences of India, Vol. 2(1): 49-55.

Montgomery, D.C. (2001) Introduction to Statistical Quality Control; New York: Wiley.

Lowry, C.A. and Montgomery, D.C. (1995) A Review of Multivariate Control Charts. II Transactions, Vol. 6(27): 800810.

Lowry, C.A., Woodall, W.H. and Rigdon, S.E. (1992) A Multivariate Exponentially Weighted Moving Average Control Chart. Technometrics, Vol. 34 (1), 46-53.

Crosier, R.B. (1988) Multivariate Generalizations of Cumulative Sum Quality-Control Schemes. Technometrics, Vol. 30(3), 291-303.

Mahmoud, M.A. and Maravelakis, P.E. (2011) The Performance of Multivariate CUSUM Control Charts with Estimated Parameters. Journal of Statistical Computation and Simulation, Vol. 1: 1-18.

Mahmoud, M.A. and Maravelakis, P.E. (2010) The Performance of the MEWMA Control Chart when Parameters are Estimated. Communications in Statistics - Simulation and Computation, Vol. 39(9): 1803-1817.

Alkahtani, S. and Schaffer, J. (2012) A Double Multivariate Exponentially Weighted Moving Average (dMEWMA) Control Chart for a Process Location Monitoring. Communications in Statistics - Simulation and Computation, Vol. 41(2):238-252.

Riaz, M. and Does, R.J. (2008) A Process Variability Control Chart. Computational Statistics, Vol. 24(2):345-368.

Costa, A.F. and Machado, M.A. (2008) A New Chart for Monitoring the Covariance Matrix of Bivariate Processes. Communications in Statistics - Simulation and Computation, Vol. 37:1453-1465.

Yeh, A.B., Lin, D.K. and McGrath, R.N. (2006) Multivariate Control Charts for Monitoring Covariance Matrix: A Review. Quality Technology \& Quantitative Management, Vol. 3(4): 415-436.

Zhang, J., Li, Z. and Wang, Z. (2010) A Multivariate Control Chart for Simultaneously Monitoring Process Mean and Variability. Computational Statistics and Data Analysis, Vol. 54: 2244-2252.

Khoo, M.B., The, S.Y. and Wu, Z. (2010) Monitoring Process Mean and Variability with One Double EWMA Chart. Communications in Statistics - Theory and Methods. Vol. 39(2):3678-3694.

Blazek, L.W., Novic, B. and Scott, D.M. (1987) Displaying Multivariate Data Using Polyplots. Journal of Quality Technology, Vol. 19(2):69-74. 
Therrien, C.W. (1989) Decision Estimation and Classificaton, An Introduction to Pattern Recognition and Related Topics. John Wiley \& Sons.

Hotelling, H. (1947) Multivariate Quality Control Illustrated by the Air Testing of Sample Bombsights. Techniques of Statistical Analysis, 111-184.

Seber, G.A.F. (1984) Multivariate Observations. Wiley: New York.

Tracy, N.D., Young, J.C. and Mason, R.L. (1992) Multivariate Control Charts for Individual observations. Journal of Quality Technology, Vol. 24:88-95.

Bersemis, S., Psarakis, S. and Paranetos, J. (2007) Multivariate Statistical Process Control Charts: An Overview. Qual. Reliab. Engng. Int, Vol. 23:517-543.

Jackson, J.E. (1991) A User Guide to Principal Components. Wiley: New York.

Fukunaga, K. Introduction to Statistical Pattern Recognition. (2nd ed.). Boston: Academic Press, 1990.

R Development Core Team (2008). R: A language and environment for statistical computing. R Foundation for Statistical Computing, Vienna, Austria. ISBN 3-900051-07-0, URL http://www.R-project.org. 